\title{
Homolateral ataxia and crural paresis: A vascular syndrome ${ }^{1}$
}

\author{
C. M. FISHER AND MONROE COLE \\ From the Neurology Service of the Massachusetts General Hospital and the Department of Neurology, Harvard \\ Medical School, U.S.A.
}

In the past few years, at the Massachusetts General Hospital, we have observed 14 patients having suffered strokes with an unusual neurological deficit in which the arm and leg on the same side showed a combination of severe cerebellar-like ataxia and pyramidal signs. The cases so strongly resembled one another that the clinical picture may be regarded as a cerebrovascular syndrome.

In a typical case weakness of the lower limb, especially the ankle and toes, and a Babinski sign were associated with a striking dysmetria of the arm and leg on the same side. The neurological examination was otherwise almost normal. The only available neuropathological examination was indecisive because of the great number and complexity of the lesions which had accumulated by the time the patient died. The site of the responsible lesion is therefore at present uncertain and indeed it has been difficult to select an anatomical locus which satisfactorily accounts for the neurological signs. Consideration of these cases requires discussion of the more general topic of appendicular ataxia resulting from supratentorial hemispheral lesions, a puzzling clinical finding. Furthermore it needs to be emphasized that in cases of stroke many kinds of incoordination are often encountered which are difficult or impossible to categorize accurately.

\section{FIVE TYPICAL CASE HISTORIES}

CASE 1 The patient, a man aged 44, while walking one afternoon noted that the right leg 'acted queerly'. Ten minutes later while seated he found that on lighting a cigarette the right arm overshot its target. As he walked 200 yards to his physician's office, the right leg tended to fall out laterally as if the knee were 'wrong' and threatened to buckle. As he waited, turning the pages of a magazine was incoordinate. After a 30-minute examination, as the patient was putting on his shoes, all symptoms cleared completely and he was able to walk with full stride. It was then approximately 50 minutes from the onset. Five minutes later the entire deficit recurred and the patient was admitted to the Massachusetts General Hospital.

${ }^{1}$ This study was aided by grant no. NB-05152 of the National Institute of Neurological Diseases and Blindness, U.S. Public Health Service.
The general examination was not remarkable except for moderate obesity and mild hypertension $(138 / 104 \mathrm{~mm}$. $\stackrel{\varnothing}{\varrho}$ $\mathrm{Hg}$ ). On neurological examination the patient was alert \& and intelligent with no speech disorder. Visual acuity was normal and the visual fields were full. The optic fundi $\vec{\circ}$ were not remarkable. The pupils were equal and reacted $\overrightarrow{\vec{H}}$ normally. The extraocular movements were full. Hori- $\vec{\omega}$ zontal nystagmus with the quick component to the left was present on left lateral gaze. Facial sensation and movements were normal. Hearing was not impaired on careful testing. There was neither dysarthria nor dys- $\infty$ phagia and the tongue protruded in the midline. Optoo kinetic nystagmus was diminished with targets moving $\frac{\vec{b}}{\infty}$ to the left.

Motor power was reduced to $6 / 10$ on dorsiflexion of th? toes and ankle on the right side. Motion was strong at the right knee and hip as it was in the other limbs.

The right arm and leg showed a severe intention tremax of a cerebellar type. On the finger-nose test wild oscills tory movements caused the patient to strike himself the face. The patient could not use the right hand for $\overrightarrow{0}$ eating. Abnormal rebound was easily demonstrateđ:. o On holding the arms out the right deviated laterally. On the heel-knee test there was a most severe dysmetria and the right thigh tended to fall laterally although weakness could not be demonstrated.

The tendon reflexes in the arms were average and equal. The right knee jerk was $3 / 4$, the left $1 / 4$. The right ankle $\stackrel{\circ}{\triangleright}$ jerk was $2 / 4$, the left $0 / 4$. The right plantar response was briskly extensor, the left flexor. Sucking and grasping reflexes were not obtained.

Sensation was normal to pin prick, temperature, vibration, position, and touch (graded hairs). Walking? was unsteady and the right leg was dragged with toescraping and circumduction as in ordinary hemiplegia. On the Romberg test the patient toppled backwards: and to the right. The patient noted the right hand felt 3 different from the left-'swollen', 'stiff', 'larger', 'as if the tendons are tight'.

A diagnosis of cerebral thrombosis was made and anti- $₹$ coagulant therapy was started. The ataxia of the right 응 upper extremity decreased gradually and at the end of one $D$ week the patient could use it for cutting his meat and o eating. Nystagmus was still present on gaze to the left. The right thigh fell laterally on attempting the heel-knee $N$ test; on hip flexion the force of contraction fluctuated $\mathrm{N}$ on different examinations. Dorsiflexion of the great toe $\mathcal{N}$ was still weak and the patient said he could exert a better $\mathrm{C}$ 
force if he turned his head to one side. The tendon reflexes were unchanged and there was still a right Babinski sign. On the Romberg test the patient swayed but remained standing. The gait was lurching and the right toe still scraped the floor. After five months, recovery was judged to be complete. There were no further incidents in the next three years.

CASE 2 The patient, a man aged 57, got up during the night to go to the bathroom and staggered badly. On awakening in the morning he noted dragging of the left leg, tingling of the left arm and leg, and a 'tremor' of the left hand.

Examination later in the day disclosed an alert patient in full charge of his faculties. The cranial nerves were not remarkable except for slight inequality of the pupils (right $2 \mathrm{~mm}$., left $2.5 \mathrm{~mm}$.). Speech was clear. There was no facial weakness. The left arm was slightly weak in all parts $(9 / 10)$. The left leg was definitely weak with power at the hip of $6 / 10$, knee $4 / 10$, dorsi- and plantar-flexion at the ankle $3 / 10$ and at the toes $0 / 10$. There was a most striking wild cerebellar ataxia of the left arm and leg on all tests of coordination. The tendon reflexes were slightly increased on the left. The left plantar response was absent, the right was flexor. Sensation was intact. Tone was slightly increased in the left upper extremity. The rebound phenomenon was prominent in the left arm. The blood pressure was $180 / 105 \mathrm{~mm}$. Hg. The spinal fluid was normal. There had been no headache.

Great improvement took place in two weeks and a left Babinski sign had become evident. Three months later there was very slight cerebellar ataxia in the left extremities and a left Babinski sign. There was no further neurological trouble in the next two years.

CASE 3 A man, aged 78, on finishing supper at 6 p.m. arose from the table and fell to the right to the floor. He was able to get to his feet and walked home a few hundred yards but had to push himself to the left to correct an inclination to veer to the right. The next day he walked a mile satisfactorily. Two days later he was worse again and could scarcely stand, tending to wobble and fall to the right. He was admitted to the Massachusetts General Hospital. The right leg distal to the mid-calf felt numb or tight for a few hours and then returned to normal. There had been no dizziness, diplopia, numbness of the face, tinnitus, or deafness.

Close questioning disclosed that the patient for about three years had been 'unsteady' on his feet, described as a sensation of staggering, lurching, or veering. Neurological examination three months previously had shown no ataxia and the patient could walk heel-to-toe.

Five years previously the patient had suddenly suffered severe upper thoracic back pain radiating downwards into the legs which became numb and collapsed under him, causing him to fall to the ground. There was abdominal epigastric pain and the patient became ashen. He was 'a bit hazy mentally', and at night usually was delirious. Radiographs of the chest showed the recent development of a bulbous enlargement of the transverse portion of the arch of the aorta. Recovery from the illness was complete.

On examination there was little abnormal except for stance. The patient was alert and pleasant; memory was mildly impaired. The fundi were not remarkable. The visual acuity was good and the visual fields were full. The pupils could not be tested because of pilocarpine therapy for glaucoma. The extraocular movements were full except for upward gaze. Nystagmus was not present. There was no cranial bruit. The arms and legs were strong. Rapid alternating movements were performed well bilaterally. Cerebellar ataxia of the mildest degree was found in the right leg but there was none in the left. Sensation in the arms was intact for pin prick, vibration, touch, position, and temperature. In the legs vibration sense was almost absent below the knees and pin prick sensation was impaired over the dorsum of both feet. Position and light touch sensation were intact. The tendon reflexes were slightly brisker on the right, especially in the arm. The right knee jerk was $3 / 4$, the left $2 / 4$. Both ankle jerks were absent; the plantar response was flexor bilaterally. On standing with the feet together the patient promptly fell to the right; closing the eyes made no difference. He was unable to walk heel-to-toe. When sitting on the bed with the feet dangling he slowly toppled to the right. The blood pressure, which had been $180 / 120$ $\mathrm{mm}$. $\mathrm{Hg}$ four months before, was $150 / 94 \mathrm{~mm}$. Hg. All pulses were absent at the ankles.

Cerebral thrombosis was diagnosed and anticoagulant therapy begun. The patient improved slowly. On the thirteenth day he could walk better but on standing with the feet together he still toppled to the right and on walking leaned far to the right. On the eighteenth day on getting out of bed in the morning he fell to the floor because of incoordination of the right leg. The next day he was worse and had little control over the right leg, veered to the right again, and could hardly walk. On the twentieth day the right leg was weak. Control of the right arm was impaired and he could not write his name or feed himself. He could still wind his wristwatch. At times he noted a slight thickness of the tongue in talking and for a while the right arm was numb. The right foot distal to the ankle felt as if bandaged. There was no diplopia or headache. Examination now showed a marked cerebellarlike ataxia of the right arm. The finger-nose test disclosed a gross wavering dysmetria chiefly at the shoulder and not explained by weakness. Rapid alternating movements of the distal parts, however, were carried out well. Power in the right hand was approximately normal, possibly reduced to $9 / 10$ at the shoulder and elbow. The handdynamometer readings were right 80 , left 70 . The patient could not handle a cup of coffee. There was a mild right ptosis. No dysphagia or dysarthria was noted although the patient thought his articulation was not clear. There was minimal flexion and extension of the toes and dorsiflexion of the ankle. At times good force was exerted momentarily only to fail immediately as in case 5. Plantar flexion of the ankles was 5/10. Motor power at the right hip was $5 / 10$ to $7 / 10$ but the patient had difficulty in making a coordinated effort. With the leg straight he could hold the heel off the bed for at least 10 seconds. On the toe-finger test there was a gross swinging ataxia. The heel-knee test and heel-tapping could not be done even when the thigh was supported by the examiner. Muscle tone was normal. 
The tendon reflexes in the right arm were exaggerated, the right knee jerk was much brisker than the left and the right plantar response was consistently extensor. Sucking and grasping reflexes were absent. The patient could not stand or walk and toppled to the right. On sitting the patient fell to the right. The blood pressure was 160/100.

Improvement took place in the next two weeks. The ataxia of the right arm almost disappeared. There was still weakness of the right lower extremity at all joints and all movements were grossly ataxic. It was difficult to decide if the incoordination was attributable to the weakness. When heel tapping was carried out with the examiner supporting the right, gross dysmetric ataxia still persisted. The tendon reflexes remained brisker on the right. The patient could support his weight on the right leg but could not walk by himself. At the end of two months he walked pushing a chair before him. Strength on extension and flexion of the knee was graded at $7 / 10$ to $8 / 10$. Yet he said, 'I have to get strength in that knee. It wobbles. It's weak. It quivers'. The patient still toppled if he sat or if he stood with the feet 4 inches apart. The plantar response on the right was flexor when the sole was stimulated medially, extensor on lateral stimulation; the left response was always flexor. On eliciting the left knee jerk, brisk adduction of the opposite thigh occurred. This response was not evoked on tapping the right side. At the end of seven months he could shuffle quickly along using the cane. The right upper extremity was normal. The right leg was still ataxic and weak; on urging, however, and repeated trials the power at each joint became almost normal for a brief time. He could stand with his feet together but on closing the eyes he lost his balance after swaying for 30 seconds.

This case exemplifies well the difficulty in interpreting the clinical signs. Inability to stand or sit and toppling to the right initially suggested a lesion of the right superior cerebellum. As the deficit increased the new signs seemed cerebellar in origin but when severe weakness appeared in the right lower limb the possibility arose that the ataxia was a manifestation of weakness. This was unlikely, however, because the instability persisted while sitting and dysmetria of the lower extremity was present even when the thigh was supported. The pyramidal signs on the right were never prominent but the tendon reflexes were clearly exaggerated and the plantar response was extensor for several weeks. Spasticity and clonus did not appear. Nystagmus was recorded only once. Unlike the other cases the most persistent weakness in the leg involved the hip flexors, although dorsiflexion of the great toes and ankle was originally the weakest movement. The sensory manifestation was chiefly subjective and evanescent, tightness of the foot and numbness of the right hand. Ptosis was noted as in case 4 . There was a hint of dysarthria at times. The absence of any dizzy feeling in the head was remarkable. The patient was right-handed yet there was no dysphasia.
The patient lived three more years in which time he had at least four more strokes, the final one being a massive left sensorimotor hemiplegia caused by a recent occlusion of the right internal carotid artery. There were at least 11 old lesions in the brain, most of them taking the form of lacunes. The lesions on the left side of the brain, which might account for the patient's clinical picture described above, were as follows: a $4 \times 2 \mathrm{~mm}$. lacune in the middle cerebellar peduncle, a $2 \mathrm{~mm}$. cavity in the mid-frontal white matter, a $5 \mathrm{~mm}$. cavity in the anterior frontal white matter, a $1 \mathrm{~cm}$. trabeculated slit in the mid-putamen, scattered small cortical infarcts in the border zone between the anterior and middle cerebral artery territories, and finally a large 9 by $5 \mathrm{~mm}$. cavity in the most posterior part of the internal capsule far superiorly adjacent to the corona radiata. The cerebellum was not involved. The suggestion will be advanced below that the large lesion in the internal capsule was possibly responsible for the ataxia and crural paralysis.

CASE 4 One afternoon this patient (right-handed), a man aged 84, got up from his chair and collapsed because of weakness of the right leg. He was admitted to theo hospital where the right leg was found to be completelyळ paralyzed and the movements of the right arm were $\mathbb{}$ totally lacking in control. There had been a pins and $\mathcal{Q}$ needles feeling in the right leg at the onset. No warning $\mathbb{D}$ had been noted but for 'some time' the right leg or knee? had given out intermittently. There had been no dizziness, dysarthria, diplopia, dysphagia, incontinence, or headache. For several months he had noted numbness or a sleeping feeling of the toes of the left foot and intermittent claudication. Diabetes mellitus had been diagnosed 56 years before. The patient was first examined by us two weeks later when considerable improvement had already occurred.

Examination on the fifteenth day of illness disclosed an alert elderly man with good memory. The cranial nerves were not remarkable except for a trace of right facial weakness on smiling. The visual fields were full. The pupils were equal and reacted to light. The extraocular movements were full without nystagmus. The tongue and palate moved normally. There was no dysarthria or dysphasia. The carotid pulsations were strong and no bruit was heard in the neck or over the eyes.

Motor power in the right arm was approximately normal. Rapid alternating movements of the fingers and forearm were slowed. There was a moderately severe cerebellar type of ataxia with clumsy oscillatory inaccurate movements on the finger-nose test. The patient could not use his right hand for taking a drink or handling his eating utensils. On holding the upper limbs outstretched the right drifted downwards. The right lower extremity showed mild weakness of the flexors of the hip and knee and almost total paralysis of the ankle and toes. On the heel-knee and toe-finger test there was a moderately severe jerky cerebellar ataxia, possibly influenced by the weakness present at the hip. Tone was questionably in- 
creased in the right limbs. Motor power and coordination of the left limbs were normal.

Sensation in the arms was normal except for light touch which was decreased on the right hand and forearm. In the legs sensation for vibration, touch, and pin prick was diminished bilaterally; the appreciation of temperature and position was intact.

The tendon reflexes were brisker in the right limbs and there was a right Babinski sign. The jaw jerk was minimal. Sucking and grasping reflexes were absent. The patient was unable to stand by himself, failing to adjust his centre of gravity to the position of his leg.

All pulses were absent at the ankles. The blood pressure was $140 / 64 \mathrm{~mm}$. Hg. Although the early course of the illness was not personally observed the picture after two weeks was similar to that of the other patients. Nystagmus was missing and light touch may have been impaired in the right arm. The absence of dysarthria and dysphasia was noteworthy.

CASE 5 On stepping out of a hot bath one morning the patient, a man aged 66, staggered and almost fell. This unsteadiness lasted only a few minutes. On getting out of the car at his office one hour later he staggered again and could scarcely stay upright. Within a few minutes he improved and was able to walk to his office. One hour later the ataxia returned and although lessening shortly thereafter it did not clear completely. At noon while being examined by his physician he lurched heavily and would have fallen had he not been caught. On the Romberg test the patient fell to the left. During the afternoon there was definite improvement but at supper time he again could scarcely stand. While sitting he felt perfectly comfortable and there was no dizzy feeling in the head. There was no numbness, diplopia, dysarthria, or weakness. He described the difficulty in walking as though the left knee unlocked or bent with a jerk threatening to send him to the floor.

The patient had had a brief stroke involving the left side four years before. On that occasion three brief attacks of numbness and tingling of the left arm and leg had occurred over a period of six hours at which time the symptoms persisted. Examination showed minimal weakness of the left leg involving the flexor groups at the hip, knee, and ankle. The left heel-knee shin test was performed inaccurately. The left ankle jerk was brisker than the right and the left plantar response was extensor. Distal to the left knee, sensation was diminished minimally for pin prick and touch, severely for position and vibration. In one day all signs cleared except the left Babinski sign which became equivocal a few days later. The patient received anticoagulant therapy at that time for eight months.

On examination the patient was clear mentally. The cranial nerves were not remarkable except for an inconstant horizontal nystagmus on gaze to the left and a slight droop of the left upper eyelid which had been present since the age of 12 . Visual acuity was excellent and the fields were full on confrontation. The pupils were equal and reacted to light and on convergence. The extraocular movements were full. There was no facial weakness or dysarthria and the tongue protruded in the midline.
Hearing had been increasingly impaired bilaterally for 20 years or more; this was familial. The carotids pulsated well and there was no intracranial bruit.

Motor power was excellent everywhere except for dorsiflexion of the left toes and ankle which was moderately weak. Occasionally brief contractions of almost normal strength were possible. There was a cerebellarlike ataxia of moderate degree in the left upper and lower extremities, most evident on the finger-nose, toe-finger, and shin-tapping test. Sensation to pin prick was slightly decreased over the entire left side, including the upper part of the face, and cold stimuli were slightly cooler on the left side. These changes were attributed to the previous stroke. Position sense, vibration, and light touch were equal on the two sides. The tendon reflexes were brisker on the left at triceps, biceps, brachioradialis, knee, and ankle. The left plantar response was extensor, the right flexor. No sucking or grasping reflexes were obtained. On standing with the feet together the patient fell to the left. On walking he also tended to topple to the left. The blood pressure was $180 / 100 \mathrm{~mm}$. Hg.

The cerebrospinal fluid was clear, under a pressure of 180 , acellular, and contained $87 \mathrm{mg}$. protein per $100 \mathrm{ml}$. The blood cholesterol was $358 \mathrm{mg}$. per $100 \mathrm{ml}$. The sedimentation rate was $28 \mathrm{~mm}$. in one hour.

After four days very gradual improvement took place. Anticoagulant therapy was maintained. On the fifth day the patient still complained that the left knee tended to jerk into flexion producing instability. $\mathrm{He}$ attributed this to weakness but careful motor testing showed normal power. On the Romberg test swaying occurred but the patient did not topple. Nystagmus on gaze to the left and cerebellar ataxia in the left arm and leg were still evident. The ataxia was greater in the leg than in the arm. The tendon reflexes on the left were a little brisker and the left plantar response remained extensor. At the end of four weeks nystagmus, ataxia of the left upper extremity, and weakness of dorsiflexion of the left great toe had cleared. Ataxia of the left lower extremity gradually lessened in the ensuing months and at the end of one year only a minimal residuum of instability on walking remained.

In this case the previous occurrence of a left-sided stroke involving the leg more than the arm complicated the picture. That attack, although evanescent, was associated the first day with incoordination and weakness. The plantar response was extensor or equivocal for some months thereafter. In the present illness a cerebellar-like ataxia of the left extremity (leg greater than arm) impaired stance and gait. Nystagmus, weakness of the dorsiflexors of the great toe and ankle, and mild pyramidal signs bring the case well within the scope of the syndrome under discussion. Since both strokes involved the same parts it is likely that approximately the same cerebral region was affected. The occurrence of a sensory deficit in the first stroke might assist in determining the site of the lesion, although the combination of sensory and cerebellar deficits on the same side of the body still poses a major problem in localization. 


\section{DISCUSSION}

THE CLINICAL PICTURE Thirteen of the 14 patients were men. The ages ranged from 44 to 84 . Twelve of the patients were hypertensive, the other two had blood pressures of $140 / 64$ and $150 / 92 \mathrm{~mm}$. $\mathrm{Hg}$. Preceding the stroke, half of the patients had one or more brief transient ischaemic attacks occurring over a relatively short period of a few hours. In the others, the stroke came on abruptly without warning. In some the paralysis occurred in the night, in others during the morning or afternoon.

In the typical case at the onset there was difficulty in walking (imbalance, staggering, weak leg, 'leg acted queerly') and incoordination of the arm on the same side as the affected leg. At least eight patients also had transient paraesthesias (swollen stiff hand, tingling of the arm and leg, leg felt bandaged, a tight feeling in the leg, tingling in the leg, tingling in the opposite leg for a few hours followed by tingling in the face, arm, and leg on the affected side for 24 hours, tingling in the arm, numb feeling over the dorsum of one foot). Headache did not occur and there was no record of diplopia, vertigo, incontinence, or mental change.

On examination there was a gross oscillatory ataxia of the limbs on the affected side. The leg was weak, more distally than proximally, and in some cases only the toes and ankle were involved. The arm showed little or no weakness. The face was spared and usually there was no dysarthria. A Babinski sign was always present on the involved side and the tendon reflexes were enhanced. Sensation was intact in all but one case. Walking was impossible, or almost so, without support. Two patients had nystagmus. Residual deficits from former strokes complicated the picture in two cases. The electroencephalogram done in five cases was normal.

THE VASCULAR LESION The pathological process is almost certainly cerebral thrombosis associated with atherosclerosis and hypertension. The stuttering onset, the occurrence of transient ischaemic spells, and in some cases the prompt recovery are all in favour of thrombosis with infarction. Haemorrhage and embolism can almost be excluded as possibilities. If the deductions to be made in the following paragraphs are correct, the site of the vascular occlusion lies in the lenticulo-striate artery or one of its posterior branches, or in a penetrating branch of the anterior cerebral artery running to the corona radiata.

TREATMENT AND PROGNOSIS We have used anticoagulant therapy (Coumarin derivatives with or without heparin) and although the clinical course in most cases was favourable we are unable to judge from our data whether such treatment was beneficial or not.

Recovery was almost complete in 12 of the 14 cases and none progressed to a severe hemiplegia or disabling aphasia. On this basis, it can be concluded that the syndrome is relatively benign and that arteriography and any therapy carrying a significant risk are to be avoided. During convalescence several of the patients complained of an instability of the knee.

INTERPRETATION OF SIGNS The weakness of the lower extremity can be attributed to a unilateral lesion of the cortico-spinal or pyramidal system; in all cases it was associated with a Babinski sign and in several cases there were increased tendon reflexes. Holmes (1922) found that lesions of a cerebellar hemisphere result in distinct weakness of the arm but not of the leg and a Babinski sign was never associated. We have tested motor power in a series of patients with severe cerebellar ataxia without pyramidal signs and in none was there weakness of the flexors of the toes, ankles, or hips.

The nature of the ataxia of the limbs is much less certain. The semi-rhythmic oscillation of the limbs during action, increasing on nearing the target, the dysmetria and dys-synergy on rapid alternating movements, and the presence of the rebound pheno- $\frac{D}{0}$ menon resulted in all observers labelling the inco ordination as cerebellar in type. Nonetheless the unusual combination of pyramidal and cerebellate signs on the same side of the body as the result of $a$ single stroke made it necessary to question the validity of designating the disorder as truly cerebellar. In the absence of a crucial diagnostic laboratory criterion of cerebellar dysfunction, one must be satisfied with the clinical impression that the disturbance of coordination was highly reminiscent of a cerebellar deficit.

In addition most of the patients showed a positive Romberg test, toppling towards the affected side, an imbalance which was not accounted for by weakness of the leg.

THE SITE OF THE LESION If the lesion found in the upper part of the posterior limb of the internal capsule in case 3 had been clearly established as the basis of the syndrome, deliberation in the matter of localization could be brief. We would have to conclude that a capsular lesion involving the corticospinal fibres to the opposite toes and ankle can also evoke cerebellar-like signs in the opposite arm and leg. The neurological picture in case 3 , however, was not as straightforward as in several other cases and the clinico-pathological correlation was far from 
satisfactory. Localization of the responsible lesion in the internal capsule can therefore be only a tentative suggestion.

If first we set aside for the moment the proposition that the syndrome is the result of a supratentorial lesion, how might an infratentorial lesion explain the symptoms? If a lesion lay bilaterally in the basis pontis, the unusual combination of symptoms might result but it would be unlikely that such a lesion on one side would produce only pyramidal signs and on the other only cerebellar signs. Another possibility is a unilateral lesion of the basis pontis affecting both the pyramidal system and the pontine nuclei or their processes destined to cross to the opposite middle cerebellar peduncle. This theoretically would produce a combined pyramido-cerebellar disturbance in the same limbs but a clear example of this phenomenon has never been reported. Bilateral cerebellar signs would be more likely. A unilateral lesion lying at any point in the brain-stem between the midmedulla and the midbrain would ordinarily be expected to produce crossed cerebellar ataxia and pyramidal signs. What is more, in our cases there were few if any associated brain-stem signs. The peripheral cranial nerves were spared and ocular motor function was intact. Two of the patients had a weak horizontal nystagmus, a somewhat indefinite finding but one which should not be dismissed too readily.

If theresponsible lesion were in the upper midbrain or subthalamic region it would be possible to involve the superior cerebellar peduncle after it has crossed the midline, and the cerebral peduncle lying immediately anterior to it, producing a combined cerebellopyramidal disturbance on the opposite side. In our cases there were no signs of midbrain involvement such as a third nerve palsy, adventitious movements, or impairment of memory. In case 3 there was no midbrain lesion at necropsy. The description of sensory symptoms and the objective sensory findings did not suggest any anatomical pattern of involvement and were of no help in localization. Of the eight patients with paraesthesias, the face was involved in only one. This brief discussion points to the difficulties of finding a suitable locus in the brain-stem.

To return to a consideration of a supratentorial site for the lesion such a localization would have the advantage of readily accounting for the homolaterality of the pyramidal and cerebellar signs. Although 'cerebellar' ataxia as the result of hemispheral (or capsular) lesions seems to be uncommon the literature contains several references to its occurence, not only with bifrontal tumours but with other lesions as well.

A paracentral ataxia was described by Claude and Lhermitte (1916) in three patients with bilateral war wounds of the pate who showed a spastic paraparesis combined with prominent cerebellar signs in the legs. The gait was wide-based, slow, festinating, uncertain, drunken, and the patient had to look at the floor. Rombergism was obvious. The legs showed dysmetria and asynergy. Sensation was intact. Two of the three had in addition a less severe dysmetria of the left arm. Two further patients with similar wounds had much the same clinical signs except that sensation in the legs was severely disturbed. Cerebellar signs were found only with bilateral lesions in the paracentral region and not with head wounds at other sites. The authors concluded that cortical lesions can produce cerebellar as well as pyramidal signs, probably by interrupting the corticopontine paths running via the basis pontis to the opposite cerebellar hemisphere. Benisti (1918) reported another patient with identical symptoms. These cases, in so far as they demonstrate that a purely motor deficit in the legs can be associated with cerebellar ataxia, are directly relevant to the syndrome under discussion, in which the lower limb is primarily affected. Furthermore it has been our experience that in ordinary hemiplegia, presumably of supratentorial origin, it is not uncommon to find on the heel-knee, toe-finger, and heel-tapping tests a moderate incoordination of the leg which is difficult to distinguish from cerebellar ataxia and this might be taken as a hint that cerebellar symptoms may be closely allied to pyramidal disorders affecting the leg. Even in some cases of paraparesis resulting from spinal cord disease the legs may show a gross dyssynergy and dysmetria not easily attributable to spasticity or weakness.

Foix and Thevenard (1922) described a patient with a verified tuberculoma of the right posterior paracentral region who showed cerebellar signs in the left extremities. In the lower extremity the heelknee test elicited dysmetria and trembling. In the upper extremity movements were clumsy, and the finger-nose test showed trembling similar to that seen in multiple sclerosis. Adiadochokinesis was present. On the test of prehension, the fingers were spread excessively. The tendon reflexes were increased in the left lower extremity and the left plantar response was not as clearly flexor as the right. There was no ideomotor apraxia. The affected limbs were hypotonic and the tendon reflexes were pendular. Sensation was intact except for the sense of position in the left great toe. On walking the left leg was flung forward more strongly than the right. Equilibrium was normal. On walking on all fours, dysmetria and asynergy of the left arm and leg were clearly present.

Alajouanine and Lemaire (1925) described a patient with a paracentral tumour (unverified) who, 
on examination, showed clumsiness of fine finger movements and oscillation and decomposition of movement in the heel-knee and finger-nose tests. Hypotonia was noted but the knee jerks were not pendular and terminal tremor was not present. On standing with the eyes closed or walking tandem the patient fell to the right. On gaze to the left intermittent nystagmus appeared. Hearing was normal. Any weakness was questionable and involved the hip flexors.

In cerebrovascular disease, involvement of the paracentral region would indicate occlusion of the anterior cerebral artery. Yet in some 15 such cases examined personally and proved by arteriography or at necropsy, the syndrome under discussion has failed to appear. In case 3 the brain showed scattered cortical lesions in the border-zone region between the anterior and middle cerebral territories but the paracentral lobule was intact and the anterior cerebral arteries were patent. Nevertheless, a lesion in the territory of the anterior cerebral artery must for the present remain one of the possible explanations of the syndrome.

A case closely resembling our group of cases was mentioned by Nicolescu, Cretu, and Demetresco (1920) in their discussion of the interior cerebral artery syndrome. A 78-year-old man, as the result of a small stroke, developed an appreciable motor deficit of the right lower limb with lively reflexes on that side but no Babinski sign. Movements were brusque and clumsy, the knee jerks pendular, and the gait a mixture of clumsiness and motor deficit. There was no apractic or sensory disturbance. No pathological study was made. The authors attributed the syndrome to a lacune involving the cortico-pontine cerebellar fibres in the frontalparacentral region above the anterior limb of the internal capsule.

The 'frontal ataxia' of Bruns and other pseudocerebellar signs with tumours and injuries of the frontal lobe have long been held by many authors to be the result of involvement of the fronto-pontine radiation. In the original cases of Bruns (1892) the disturbance was predominantly one of stance and gait with complete loss of balance and inability to walk without support (atasia-abasia). Incoordination of the individual limbs was not mentioned. Gerstmann (1916) described a similar picture in penetrating head injuries of the frontal region and suggested as the cause a lesion of the cortical origin of the fronto-pontine cerebellar system. His patients showed complete helplessness or great uncertainty in standing and walking, falling (the direction determined by the position of the head), nystagmus, vestibular hypersensitivity and past-pointing, but very little dys-synergy or disturbance of rapid alter- nating movements of the individual limbs. Gerst- $Z_{\mathbb{D}}$ mann and Schilder (1926) later attributed at least some of the gait disturbances of this type to motor apraxia or apraxia of gait.

Hare (1931) in 50 cases of frontal lobe tumour 0 found severe cerebellar signs (staggering gait, Rombergism, drifting of one or both arms, dysmetria in the upper extremities, hypotonia, nystagmus, ataxia in one or both lower extremities, pastpointing, dysdiadochokinesis, and asynergy of the $\overrightarrow{\vec{F}}$ pelvic muscles) in five cases and minor cerebellar $\stackrel{5}{+}$ signs in 20 further cases. Brun (1932) divided $\frac{}{\circ}$ cases of frontal ataxia into two groups, a bilateral $\frac{\bar{c}}{\bar{c}}$ form resulting from advanced bilateral frontal $\frac{\omega}{\sigma}$ tumours in association with raised intracranial $\varrho$ pressure, and a more specific type of ataxia always unilateral and involving the opposite leg. Examina- $\vec{\circ}$ tion in the second group could be normal in the early stages except for walking a straight line heel-to-toe, $\vec{\omega}$ when the affected foot moved more or less uncertainly and was not placed squarely in front of the other; and at times the legs might be crossed. The i author was inclined to attribute the unilateral 9 disturbance to interruption of the fronto-pontine fibres to the opposite cerebellar hemisphere. Othe $\vec{P}$ had also noted faulty placing of the affected foot i⿱ walking: for example, one foot often trod on the other (Bruns, 1892), one foot crossed in front of the other (Gerstmann, 1916), and the patient put the 을 affected foot in the position the other foot was assume in the next step (Gerstmann and Schilde 1926). Frazier (1936) in a large series of front $\vec{\theta}$ lobe tumours found frontal ataxia in $49 \%$ of cases. Gordon (1934) concluded that frontal ataxia differed $\square$ from the cerebellar variety in that the rebound phenomenon, hypotonia, and pendular knee jerks were missing in the former.

These descriptions of frontal ataxia are almost $\stackrel{\varrho}{\varnothing}$ exclusively from cases of tumour or trauma, not $\stackrel{\varrho}{\Rightarrow}$ reliable conditions on which to base inferences $\overrightarrow{\overrightarrow{0}}$ concerning localization of function. We were unable to find any reference to the occurrence of 'frontal ataxia' in cases of pathologically verified infarction of either the cerebral cortex or the tracts descending therefrom in the corona radiata or internal capsule. It should be pointed out that in case 3 the frontopontine tracts, at least as depicted in anatomical texts, were apparently spared. Also these tracts are $ᄋ$ not immediately adjacent to the cortico-spinal foot $₹$ and leg fibres in thecerebral hemisphere and the weak- 을 ness of the foot would fail to be accounted for. $A D$ strictly frontal location is unlikely because of the absence of sucking and grasping reflexes, the intact mentality, normal limb postures, and control of $\%$ sphincters.

It should be pointed out that although the fronto- N్ల 
pontine system occupies the anterior limb of the internal capsule, parieto-pontine and occipitopontine fibres are said to course in the posterior limb of the internal capsule (Ranson and Clark, 1947) where the lesion lay in case 3. There appear to be several sites at which hemispheral lesions theoretically might evoke a cerebellar-like deficit.

Parietal ataxia, in which dys-synergy accompanies a post-central lesion, has also been reported. Foix, Chavany, and Levy (1927) described dysmetria, decomposition of movement, and end-point tremor in a patient with a mild hemiparesis and severe sensory loss as the result of an infarct of the parietal region. Recently on the Stroke Service of the Massachusetts General Hospital a case of parietal ataxia has been studied clinically and neuropathologically (Fisher, Appenzeller, and Descarries, 1964). The lesion, which was a well-localized infarct, involved the cortex of the post-central arm area. The clinical picture in these cases of parietal ataxia clearly differs from that of the cases being reported here.

Dwelling at further length on theoretical matters is unwarranted. In summary we are inclined to believe that a supratentorial lesion of the crural pyramidal fibres is responsible for the clinical picture. Possibly it lies in the posterior-superior part of the internal capsule as in case 3 or in the adjacent corona radiata. The ataxia would then not be attributable to a direct injury to the cerebellum or its main pathways. Only further clinicopathological studies will clarify the matter.

\section{SUMMARY}

A cerebrovascular syndrome has been described which comprises cerebellar ataxia, weakness, and pyramidal signs involving the limbs of the same side, the leg more than the arm. The pathological examination in one case showed a conspicuous infarct in the posterior limb of the internal capsule but the clinicopathological correlation was not conclusive because the brain showed many other infarcts. The respons- ible lesion is nevertheless tentatively located in the capsular-corona radiata region supratentorially. The ataxia is probably not due to direct involvement of the cerebellum or its main pathways but is related to damage to cortico-pontine connexions or to certain fibres of the pyramidal system. The underlying vascular process is probably thrombotic.

The subject of ataxia arising from supratentorial lesions has been briefly reviewed.

\section{REFERENCES}

Alajouanine, T., and Lemaire, A. (1925). Tumeur de la région paracentrale postérieure avec symptôms 'pseudo-cérébelleux'. Rev. neurol., 1, 71-75.

Benisti (née Athanasiu) (1918). Les lésions de la zone rolandique (zone motrice et zone sensitive) par blessures de guerre. Thèse de Paris, 127, Paris, Vigot.

Brun, R. (1932). Zür frage der stirnhirnataxie. Ein neues stirnhirnsymptom. Z. ges. Neurol. Psychiat., 138, 122-130.

Bruns, L. (1892). Ueber störungen des gleichgewichtes bei stirnhirntumoren. Dtsch. med. Wschr., 18, 138-140.

Claude, H., and Lhermitte, J. (1916). Les paraplégies cérébellospasmodique et ataxo-cérébello-spasmodique consécutives aux lésions bilatérales des lobules paracentraux par projectiles de guerre. Bull. Soc. Méd. Hôp. Paris, 40, 796-804.

Fisher, C. M., Appenzeller, O., and Descarries, L. P. (1964). A case of parietal ataxia. In preparation.

Foix, C., Chavany, J. A., and Levy, M. (1927). Syndrome pseudothalamique d'origine pariétale. Rev. neurol., 1, 68-76.

—_, and Thevenard, A. (1922). Symptômes pseudo-cérébelleux d'origine cérébrale, tubercule de la région paracentrale postérieure. Ibid., 1, 1502-1504.

Frazier, C. H. (1936). Tumour involving the frontal lobe alone; symptomatic survey of 105 verified cases. Arch. Neurol. Psychiat., 35, 525-571.

Gerstmann, J. (1916). Zur kenntnis der störungen des korpergleichgewichtes nach schubverletzungen des stirnhirns. Mschr. Psychiat. Neurol., 40, 354-377.

Stirnhirnerkrankung. Wien. med. Wschr., 76, 97-102.

Gordon, A. (1934). Frontal lobe lesions with cerebellar manifestations; contribution to frontal lobe as center of equilibrium. J. nerv. ment. Dis., 79, 411-422.

Hare, C. C. (1931). The frequency and significance of cerebellar symptoms in tumours of the frontal lobes. Bull. neurol. Inst. New York, 1, 532-562.

Holmes, G. (1922). Clinical symptoms of cerebellar disease and their interpretation. Lancet, 1, 1177-1182, 1231-1237, and 2, 59.65, 111-115.

Nicolescu, J., Cretu, V., and Demetresco, L. (1920). Syndrome de l'artère cérébrale anterieure. Monoplégie crurale droite avec symptomatologie cérébelleuse preponderante. Bull. Soc. med. hop. Bucarest, vol. 10. Synopsis in Rev. neurol. (1932), 1, 563.

Ranson, S. W., and Clark, S. L. (1947). The Anatomy of the Nervous System, 8th ed., Saunders, Philadelphia and London. 